\title{
Structure of the Catalytic Pore of $\gamma$-Secretase Probed by the Accessibility of Substituted Cysteines
}

\author{
Chihiro Sato, Yuichi Morohashi, Taisuke Tomita, and Takeshi Iwatsubo \\ Department of Neuropathology and Neuroscience, Graduate School of Pharmaceutical Sciences, The University of Tokyo, Bunkyo-ku, Tokyo 113-0033, \\ Japan
}

\begin{abstract}
Several single-span membrane proteins are cleaved within their transmembrane domains (TMDs) by intramembrane-cleaving proteases, although the structure of the active site executing intramembrane cleavage remains unknown. Here we use the substituted cysteine accessibility method to examine the structure of presenilin-1, a catalytic subunit of $\gamma$-secretase, involved in amyloid $\beta$ protein generation in Alzheimer's disease and Notch signaling. We show that TMD6 and TMD7 of presenilin-1 contribute to the formation of a hydrophilic pore within the membrane. Residues at the luminal portion of TMD6 are predicted to form a subsite for substrate or inhibitor binding on the $\alpha$-helix facing a hydrophilic milieu, whereas those around the GxGD catalytic motif within TMD7 are highly water accessible, suggesting formation of a hydrophilic structure within the pore. Collectively, our data suggest that the active site of $\gamma$-secretase resides in a catalytic pore filled with water within the lipid bilayer and is tapered around the catalytic aspartates.
\end{abstract}

Key words: Alzheimer's disease; intramembrane-cleaving protease; presenilin; substituted cysteine accessibility method; A $\beta$ peptide; proteolysis; structure

\section{Introduction}

Proteases hydrolyze peptide bonds using ionized water. Thus, catalytic domains of proteases are usually located within an aqueous compartment. Recently, however, a body of evidence highlights a novel group of polytopic membrane proteases with an active site buried within the membrane (Wolfe, 2006). Biochemical and cell biological analyses revealed that these polytopic proteins are directly responsible for intramembrane proteolysis, an atypical mode of cleavage occurring to scissile bonds located within the lipid bilayer. Thus, these enzymes are designated intramembrane-cleaving proteases (I-CLiPs), although the molecular mechanism of this unusual proteolytic process remains unknown.

$\gamma$-Secretase is an atypical aspartic protease that belongs to the I-CLiP family and cleaves a set of type- 1 single-span membrane proteins, including the $\beta$-amyloid precursor protein (APP), to form amyloid- $\beta$ peptides $(\mathrm{A} \beta)$ in Alzheimer's disease (Tomita and Iwatsubo, 2006). Genetic and biochemical analyses revealed

Received Aug. 20, 2006; revised 0ct. 9, 2006; accepted 0ct. 11, 2006.

This work was supported by grants-in-aid from the Ministry of Education, Science, Culture, and Sports for the 21st Century Center of Excellence Program (C.S., T.I., T.T.) and by the Program for Promotion of Fundamental Studies in Health Sciences of the National Institute of Biomedical Innovation, Japan (T.I., T.T.). We thank Drs. G. Thinakaran (University of Chicago, Chicago, IL) and A. Takashima (RIKEN, Saitama, Japan) for reagents, B. De Strooper (Katholieke Universiteit Leuven, Leuven, Belgium) for DK0 cells, T. Kitamura (The University of Tokyo, Tokyo, Japan) for the retroviral infection system, R. Kopan and M. X. Ilagan (Washington University, St. Louis, MO) for critical reading of this manuscript, and Takeda Pharmaceutical Company (Osaka, Japan) for the A $\beta$ ELISA. We are also grateful to $R$. Takikawa and Dr. R. Katayama for discussions and experiments at an early stage of this study and to our laboratory members for helpful discussions and technical assistance.

Correspondence should be addressed to either of the following: Taisuke Tomita or Takeshi Iwatsubo, Department of Neuropathology and Neuroscience, Graduate School of Pharmaceutical Sciences, The University of Tokyo, 7-3-1 Hongo, Bunkyo-ku, Tokyo 113-0033, Japan. E-mail: taisuke@mol.f.u-tokyo.ac.jp or iwatsubo@mol.f.u-tokyo.ac.jp. DOI:10.1523/JNEUROSCI.3614-06.2006

Copyright $\odot 2006$ Society for Neuroscience $\quad$ 0270-6474/06/2612081-08\$15.00/0 that $\gamma$-secretase is a high-molecular-weight membrane protein complex comprising presenilin (PS), nicastrin (Nct), Aph-1, and Pen-2. PS1 and PS2, the catalytic subunits of the $\gamma$-secretase complex, undergo endoproteolysis to generate an N-terminal fragment (NTF) and a C-terminal fragment (CTF) that harbor YD and GxGD conserved catalytic motifs within the sixth and eighth hydrophobic region (HR), respectively (Haass and Steiner, 2002). Overexpression of these four protein components reconstitutes a functional $\gamma$-secretase complex with proteolytic activity (Takasugi et al., 2003). Because of the nature of this highmolecular-weight membrane protein complex, conventional structural analysis of this atypical protease has not been feasible. Recently, a glimpse into the structure of the $\gamma$-secretase complex was obtained by single-particle analysis (Lazarov et al., 2006; Ogura et al., 2006). However, because of the lack of structural information at an amino acid level, these structures were not helpful in deciphering the molecular mechanism that underlies the ability of lipid-soluble $\gamma$-secretase to execute peptide bond hydrolysis.

Understanding the structures of HR6 and HR8, especially in their active states, is crucial for the development of novel $\gamma$-secretase inhibitors based on the molecular mechanism. Here we applied the substituted cysteine accessibility method (SCAM) to human PS1. SCAM has originally been used to identify the pore-lining residues of complex multipass membrane proteins defined by the accessibility of covalent modification by sulfhydryl reagents to cysteine residues (Karlin and Akabas, 1998; Seal et al., 1998; Kaback et al., 2001). We systematically substituted a single cysteine at every residue in HR6 and HR8 of PS1 and analyzed the accessibility of a membrane-impermeable sulfhydryl reagent, methanethiosulfonate ethylammonium (MTSEA)-biotin. To complement this approach, we performed competition assays 
using charged derivatives of methanethiosulfonate (MTS) reagent and specific $\gamma$-secretase inhibitors, and a cross-linking experiment using MTS cross-linkers. Here we propose the structure of the intramembranous catalytic pore of active $\gamma$-secretase as follows: HR6 and HR8 of PS1 penetrate the lipid bilayer as transmembrane domains (TMDs) directly facing a catalytic pore with a funnel-like structure and are involved in intramembrane proteolysis as subsites.

\section{Materials and Methods}

Materials and immunological methods. G1L3 and PNT3 antibodies, which recognize human PS1 loop and Pen-2, respectively, were described previously (Tomita et al., 1999; Hayashi et al., 2004). Anti-PS1 $1_{\mathrm{NT}}$ and PS-C3 (anti-PS1 CTF) were kindly provided by Drs. G. Thinakaran (University of Chicago, Chicago, IL) and A. Takashima (RIKEN, Saitama, Japan), respectively. Other antibodies included anti-PS1 loop (MAB5232; Millipore, Bedford, MA), antiAph-1aL (O2C2; Covance, Princeton, NJ), and anti-Nct (N-19; Santa Cruz Biotechnology, Santa Cruz, CA). $\mathrm{N}$-[N-(3,5-difluorophenacetyl)-Lalanyl]-S-phenylglycine $t$-butyl ester (DAPT) was synthesized as described previously (Kan et al., 2003). L-685,458 (Li et al., 2000) and peptide 15 (pep15) (Das et al., 2003) were purchased from Bachem (Torrance, CA) and Ito Life Sciences (Moriya, Japan), respectively. All MTS reagents (Toronto Research Chemicals, Toronto, Ontario, Canada) were dissolved in DMSO at $200 \mathrm{~mm}$ and stored at $-80^{\circ} \mathrm{C}$ until use. Immunoblot analysis, immunoprecipitation of 3-[(3-cholamidopropyl)dimethylammonio]-2-hydroxy-1-propanesulfonate solubilized lysates, and quantitation of $A \beta$ by two-site ELISAs were performed as described previously (Tomita et al., 1997; Takasugi et al., 2003; Hayashi et al., 2004; Watanabe et al., 2005; Morohashi et al., 2006).

Plasmid construction, cell culture, transfection, and retroviral infection. cDNAs encoding PS1 and APP carrying the Swedish mutation $\left(\mathrm{APP}_{\mathrm{NL}}\right)$ were inserted into pLPCX (Clontech, Palo Alto, CA) or pMXs-puro as described previously (Watanabe et al., 2005). cDNAs encoding PS1/ Cys (-) and single- or double-Cys mutant (mt) PS1 were generated using the long PCR-based protocol. All constructs were sequenced using ThermoSequenase (USB Corporation, Cleveland, $\mathrm{OH}$ ) on an automated sequencer (Li-Cor, Lincoln, NE). Maintenance of Psen1/Psen2 doubleknock-out (DKO) cells, retroviral infection, and stable infectant generation were done as described previously (Watanabe et al., 2005).

Intact cell biotinylation. Stable DKO cells were grown in 6-well plates. After washing with ice-cold PBS, cells were incubated with $1 \mathrm{~mm}$ MTSEA-biotin in PBS for $30 \mathrm{~min}$ at $4^{\circ} \mathrm{C}$. Excess reagents were removed by washing twice with PBS. Labeled cells were lysed in 1\% SDS/PBS for $3 \mathrm{~h}$, and solubilized supernatants were incubated with streptavidinSepharose (GE Healthcare, Piscataway, NJ) overnight. Biotinylated proteins were washed five times with $1 \%$ SDS/PBS, eluted by boiling in sample buffer, and analyzed by immunoblotting. For competition analyses using MTS reagents ( $2 \mathrm{mM}$ ) or inhibitors ( $1 \mu \mathrm{M}$ for L-685,458 and pep15, $10 \mu \mathrm{M}$ for DAPT), cells were preincubated with chemicals for 30 min and subjected to biotinylation by MTSEA-biotin.

Microsomal preparation and biotinylation/cross-linking experiments. Microsomal preparation was performed as described previously (Morohashi et al., 2006), with some modifications. Stable DKO cells expressing cysteine mutant PS1 were grown on two $15 \mathrm{~cm}$ dishes per single analysis. Cells were scraped into PBS and resuspended in $2 \mathrm{ml}$ of homogenization buffer [10 mm HEPES, pH 7.4, $150 \mathrm{~mm} \mathrm{NaCl}, 10 \%$ glycerol, Complete protease inhibitor cocktail (Roche Biochemicals, Indianapolis, IN)]. Cells were disrupted using a polytron homogenizer (Hitachi, Tokyo,
Japan), and nuclei and large cell debris were pelleted by centrifugation at $1500 \times g$ for $10 \mathrm{~min}$. The postnuclear supernatants were centrifuged, the resultant microsomal pellets were resuspended in $0.2 \mathrm{ml}$ of PBS in a syringe, and $1 \mathrm{~mm}$ MTSEA-biotin was added to this fraction. After a 30 min incubation at $4^{\circ} \mathrm{C}$, microsomes were centrifuged twice to wash out free MTSEA-biotin. The resultant pellets were resuspended in 1\% SDS/ PBS, incubated with streptavidin-Sepharose overnight, and analyzed as in the intact cell biotinylation experiment. For cross-linking experiments, resuspended microsomes were incubated with MTS cross-linkers ( $2 \mathrm{~mm}$ ) for $2 \mathrm{~h}$ at room temperature and directly subjected to immunoblot analysis.

\section{Results}

Cysteine mutant PS1 expressed in DKO cells fulfills the requirements for the structural analysis by SCAM

We constructed PS1 lacking cysteine [PS1/Cys $(-)]$, in which five endogenous cysteines (Fig. 1) were replaced with serines and stably expressed in embryonic fibroblasts derived from Psen $1 /$ Psen 2 DKO mice (Herreman et al., 2000). PS1/Cys(-) was endoproteolyzed and restored the maturation of Nct and the level of Pen-2 in a manner similar to wild-type PS1 (Fig. $2 \mathrm{~A}$ ). Coimmunoprecipitation analysis revealed that the PS1/Cys $(-)$ fragments interacted with other $\gamma$-secretase components (data not shown). Moreover, overexpression of PS1/Cys $(-)$ in DKO cells expressing $\mathrm{APP}_{\mathrm{NL}}$ rescued $\mathrm{A} \beta$ secretion, accompanied by a slight increase in the $A \beta 42 / A \beta 40$ ratio (Fig. $2 B$ ). Thus, all cysteines in the PS1 polypeptide are dispensable for the intramembrane cleaving activity.

To ascertain the reliability of SCAM on PS1, we then introduced a single cysteine into PS1/Cys (-) (single-Cys mt PS1) at several positions where the topology has been extensively studied by multiple methods (cytosolic location, S9C and H351C; luminal location, I114C and A192C; TMD location, S141C and S230C) (Fig. 1) (Doan et al., 1996; Li and Greenwald, 1996, 1998; Laudon et al., 2005). All of the single-Cys mt PS1 were active in 


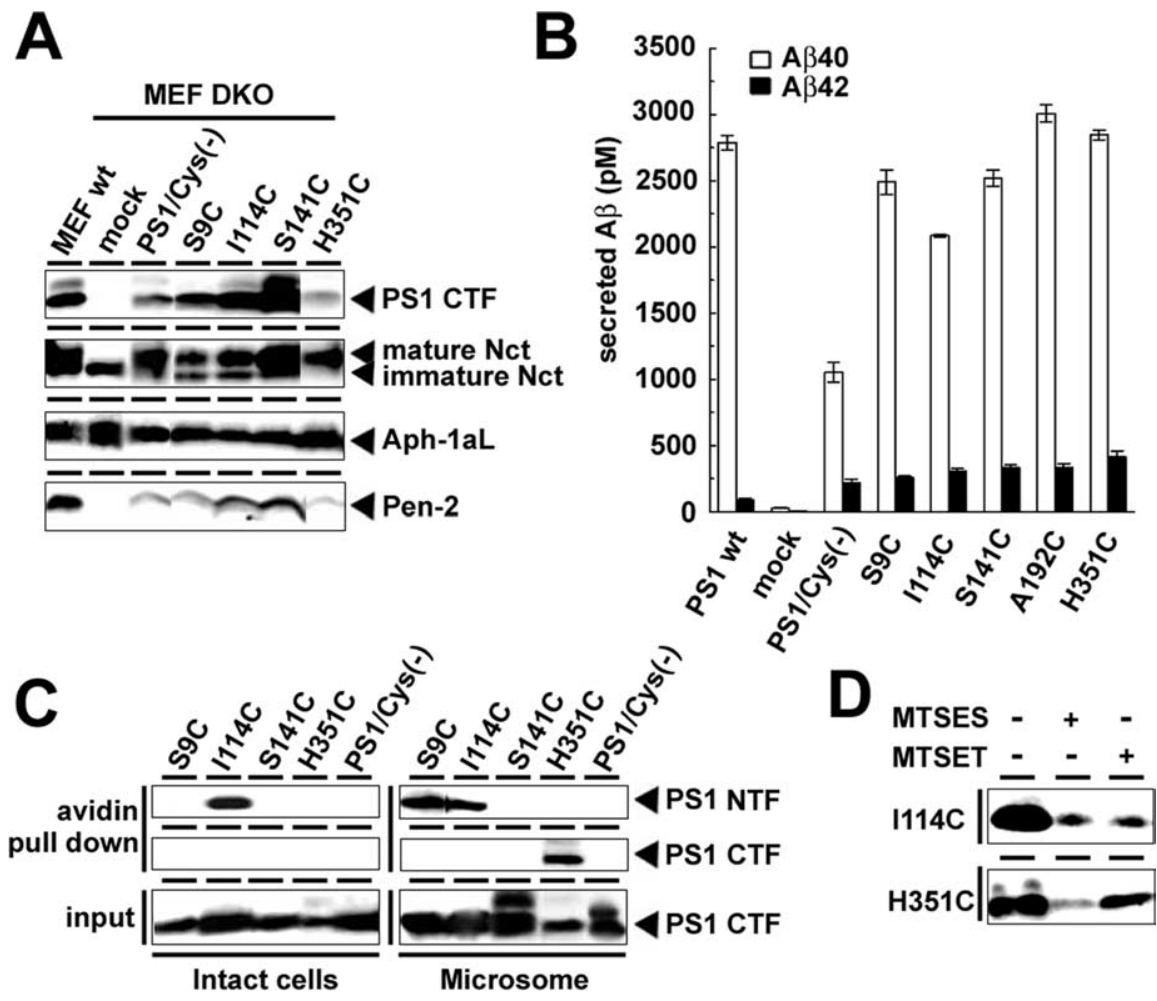

Figure 2. SCAM analysis of single-Cys mt PS1 at representative positions. $\boldsymbol{A}$, Western blot analysis of single-Cys mt PS1 stably expressed in DKO cells. Endoproteolysis of PS1 and restoration of the expression of mature glycosylated Nct and Pen-2 in all single-Cys mt PS1 are shown. $\boldsymbol{B}$, Effect of single-Cys mt PS1 on A $\beta$ levels secreted from DKO cells coexpressing APP ${ }_{\mathrm{NL}}$. Error bars indicate mean \pm SE $(n=3)$. C, Biotin-labeling experiment using MTSEA-biotin in intact cells (left) and microsomes (right). $\boldsymbol{D}$, Labeling competition by preincubation with MTSES and MTSET. MEF, Mouse embryonic fibroblast; wt, wild type.
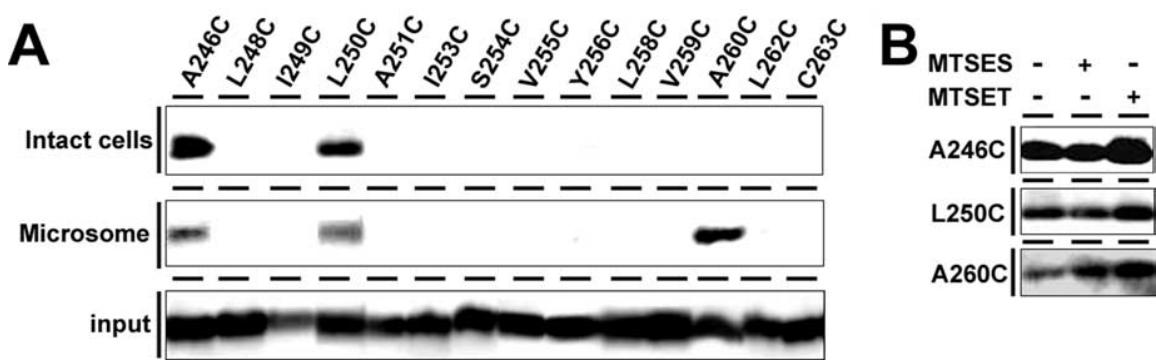

Figure 3. SCAM analysis of single-Cys mt PS1 in HR6. $A$, Biotin-labeling experiment using MTSEA-biotin in intact cells (top) and microsomes (middle). The amount of PS1 NTF in the input fraction is shown in the bottom panel. $\boldsymbol{B}$, Labeling competition by MTSES and MTSET.

terms of complex assembly, and all could reconstitute proteolytic activity in DKO cells (Fig. $2 A, B$ and data not shown). To analyze the water accessibility of the cysteine residues from the cell exterior, we labeled the surface of intact DKO cells expressing singleCys mt PS1 with a membrane-impermeable reagent, MTSEAbiotin. MTS reagents react specifically and rapidly with the sulfhydryl group of cysteine in an aqueous environment (Roberts et al., 1986). MTSEA-biotin is impermeable to the plasma membrane, and it has been used for the topological analysis of channels and transporters that are expressed on the plasma membrane (Karlin and Akabas, 1998; Seal et al., 1998; Kaback et al., 2001). Biotinylated proteins were collected by precipitation using streptavidin beads after solubilization in SDS, a procedure that disrupts the $\gamma$-secretase complex. PS1/Cys $(-)$ was never recovered in precipitated fractions. However, I114C (Fig. 2C) and A192C (see Fig. 5) were biotinylated, as predicted for residues residing within the extracellular, hydrophilic loop (HL). None of the other single-Cys mt PS1 studied here was biotinylated, indicating that no cysteine residues at the cytosolic side or within the TMD are accessible from the extracellular side using this protocol.

To examine whether the cysteine residues are accessible from the cytosolic side, we prepared membrane microsomes and subjected them to biotinylation by MTSEA-biotin, followed by a pull-down analysis (Fig. 2C). Cysteine substitutions at the residue located at the predicted cytosolic region (i.e., $\mathrm{S} 9 \mathrm{C}$ at the $\mathrm{N}$ terminus and H351C in HL6) were now labeled with MTSEA-biotin, as predicted for residues facing the cytosol and exposed to the hydrophilic milieu. In addition, the extracellular I114C was also labeled by MTSEAbiotin. This reflects the topology of membrane fractions (i.e., right-side-out, inside-out, or disrupted vesicles), which depends primarily on experimental conditions (Steck et al., 1970). Because all of the cysteine residues accessible from the cell surface were also biotinylated in our microsomal preparations (Figs. 2-4), the microsomes prepared by our protocol contained both right-side-out and inside-out membrane orientations. Notably, cysteine residues at S141 (Fig. 2) and S230 (data not shown) were inaccessible to MTSEAbiotin from either the cell surface or in the microsome preparation, indicating that these cysteine residues were embedded in the lipid bilayer.

To evaluate the accessibility of the cysteine residues, we next examined the effect of other membrane-impermeable MTS derivatives, such as the negatively charged 2-sulfonatoethyl methanethiosulfonate (MTSES) and the bulkier, positively charged 2-(trimethylammonium)-ethyl methanethiosulfonate (MTSET). The bulkiness and/or the charge of MTSES and MTSET prevent them from penetrating into narrow hydrophilic spaces because of physical and/or ionic barriers (Akabas et al., 1994; Kuner et al., 1996; Seal and Amara, 1998; Wilson and Karlin, 1998; Seal et al., 2000). Thus, if MTSEAbiotin binding is abolished by preincubation with MTSES or MTSET, we will infer that the cysteine residue was located in an open hydrophilic environment. In contrast, cysteine residues facing an aqueous but restricted environment, be it because of charge or location in an aqueous pocket within the lipid bilayer, will still be available for MTSEA-biotin in the presence of MTSES or MTSET. Differences in the competition profile by MTSES and MTSET may sometimes reflect differences in the ionic environment surrounding the cysteine residues (Seal and Amara, 1998; Wilson and Karlin, 1998). As evidence that this strategy works, we observed that the biotinylation of residues located in an accessible hydrophilic environment (I114C and H351C) was reduced by preincubation with MTSES or MTSET (Fig. 2D). 


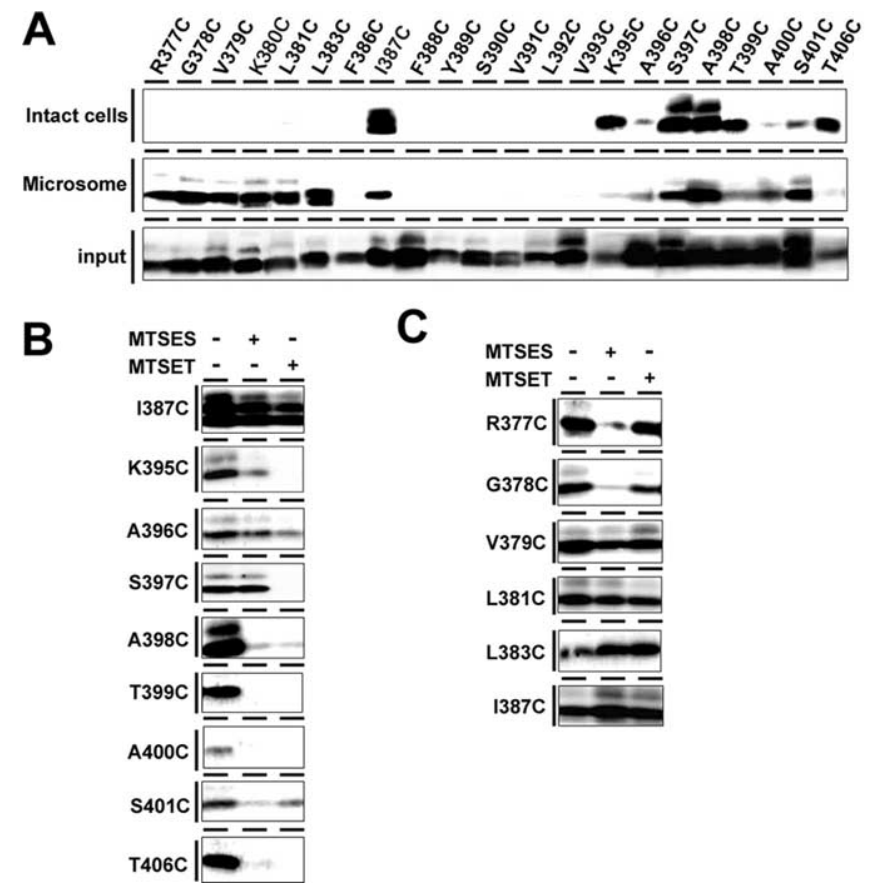

Figure 4. SCAM analysis of single-Cys mt PS1 around HR8. $A$, Biotin-labeling experiment using MTSEA-biotin in intact cells (top) and microsomes (middle). The amount of PS1 CTF in the input fraction is shown in the bottom panel. B, C, Biotin labeling of single-Cys mt PS1 around HR8 was performed after preincubation with MTSES or MTSET in intact cells $(\boldsymbol{B})$ or microsomes (C). Locations of cysteine mutations are shown on the left side of the panel.

\section{SCAM analysis of HR6 of PS1}

Most of the previous computational predictions indicated that HR6, which harbors the catalytic aspartate 257, spans the membrane as TMD6. To verify whether HR6 penetrates the membrane as a TMD and faces a hydrophilic milieu, we generated single-Cys mt PS1 at 24 amino acid residues in and around HR6 (L241-R269). Some cysteine substitutions abolished the expression of PS1 polypeptides or eliminated $\gamma$-secretase activity (i.e., L241C, T245C, V252C, D257C, V261C, P264C, L268C, R269C). Because we could not rule out the possibility that a significant structural change around the catalytic site caused PS1 to lose activity, these mutants were excluded from additional analyses (supplemental Fig. $1 A$, available at www.jneurosci.org as supplemental material). SCAM analysis of the remaining HR6 mutants in intact DKO cells revealed a strong biotin labeling of A246C and L250C, whereas no other single-Cys mt PS1 were labeled (Fig. $3 A$ ), suggesting that residues A246 and L250 are accessible from the extracellular space. However, neither MTSES nor MTSET diminished the labeling of A246C and L250C, suggesting that in contrast to $\mathrm{I} 114 \mathrm{C}$, these residues were located within a hydrophilic but narrow space inaccessible to the charged MTS reagents (Fig. 3B). We next examined the microsome labeling of singleCys mt PS1. In addition to A246C and L250C, A260C was labeled by MTSEA-biotin (Fig. 3A) even after preincubation with MTSES or MTSET in microsomal preparation (Fig. 3B). No other HR6 mutants were biotinylated by MTSEA-biotin, suggesting that other than A260, all residues within the cytosolic half of HR6 are completely inaccessible. Moreover, the inability to biotinylate A260C from the exterior of the cell supports the view that the luminal part of HR6 is exposed to a hydrophilic environment tapered at the midportion like a funnel.

\section{SCAM analysis of HR8 of PS1}

Because of the relatively weak hydrophobicity of HR8, the topology and geometry of PS1 "TMD7," wherein the catalytic aspartate D385 resides, remain controversial. To obtain information about the hydrophilicity of HR8 when incorporated in an active $\gamma$-secretase complex, we generated single-Cys mt PS1 at 29 consecutive amino acid residues in and around HR8 (R377-T406). Again, some mutants (i.e., G382C, G384C, D385C, G394C, G402C, D403C, and W404C) lost protein expression or activity and thus were excluded from additional analysis (supplemental Fig. $1 B$, available at www.jneurosci.org as supplemental material). Labeling experiments using intact cells revealed that consecutive amino acid residues from K395 to S401, and T406, were reactive with MTSEA-biotin (Fig. $4 A$ ). The labeling of all these residues was inhibited by preincubation with MTSES or MTSET, suggesting that this entire region resides in a hydrophilic environment accessible from the extracellular side (HL7) (Fig. 4B). Given that H351 locates to the cytosolic side (Fig. 2C), it is highly likely that PS1 HR8 spans the membrane as TMD7 within the active $\gamma$-secretase complex. However, unlike TMD6, residues at the luminal half of TMD7 were not labeled, suggesting that these residues do not face the water-accessible milieu. Notably, I387C was strongly biotinylated from the extracellular side, and neither MTSES nor MTSET diminished its labeling by MTSEA-biotin (Fig. $4 B$ ). This suggests that $\mathrm{I} 387$ faces a hydrophilic crevice connected to the extracellular side.

We next prepared microsome fractions from DKO cells expressing single-Cys mt PS1 of HR8 and subjected them to labeling experiments (Fig. 4A). Consistent with the previous results, residues K395 through S401, which were predicted to represent HL7 by intact cell labeling, were biotinylated, whereas the accessibility of some residues were different from those observed by intact cell labeling. Again, I387C was labeled, showing that I387 sits in an aqueous environment. Unexpectedly, all single-Cys mt PS1 from R377 to L381 and L383 were clearly biotinylated, suggesting that the cytosolic side of HR8 forms a highly water-accessible structure, unlike ordinary $\alpha$-helical TMDs facing a pore. In contrast, consecutive residues at the putative luminal side of HR8 (F388 to V393) were not labeled, consistent with the results from intact cell labeling (Fig. 4A). All residues of HR8 were clearly labeled in $1 \%$ SDS-solubilized membrane fractions, supporting the view that the luminal side of TMD7 does not directly face a hydrophilic space within the membrane that is accessible to MTSEA-biotin (supplemental Fig. 2, available at www.jneurosci.org as supplemental material). Preincubation with MTSES or MTSET diminished the labeling of R377C and G378C similarly to H351C, suggesting that these residues sit in a hydrophilic environment with unlimited water accessibility (Fig. 4C). However, the biotinylation of V379C, L381C, L383C, and I387C was not decreased by preincubation with the charged MTS derivatives. Thus, the cytosolic side of HR8 seems to reside within a hydrophilic pore, which is inaccessible from the extracellular side. Together with the results on TMD6, these data support the view that the wateraccessible space around the catalytic aspartates is narrowed like a funnel. Moreover, SCAM analysis revealed that PS1 HR8 spans the lipid bilayer forming an unusual TMD7: a portion of the luminal half of TMD7 around the catalytic aspartate participates in the formation of a hydrophilic pore, whereas access of MTSEA-biotin across the channel to the cytosolic side of TMD7 is not permitted. In contrast, the cytosolic half of TMD7, including the catalytic site with the conserved GxGD motif, is highly hydrophilic within the catalytic pore. 


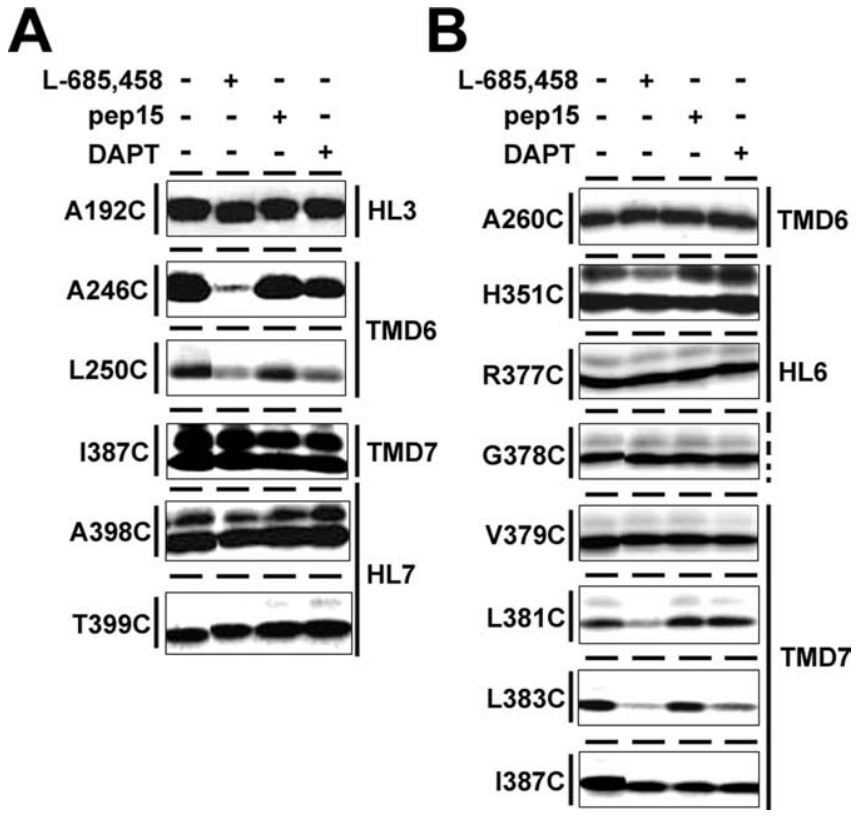

Figure 5. Labeling competition by $\gamma$-secretase inhibitors. Biotin labeling of single-Cys $\mathrm{mt}$ PS1 was conducted after preincubation with $L-685,458$, pep15, or DAPT in intact cells $(\boldsymbol{A})$ or microsomes $(\boldsymbol{B})$. Locations and predicted topology of cysteine mutations are shown on the left and right sides of the panel, respectively.

\section{Reactivity of substituted cysteines in the presence of $\gamma$-secretase inhibitors}

Transition-state analog-type aspartyl protease inhibitors can be modified to demonstrate direct binding to the PS fragments, suggesting that PS harbors the catalytic site of $\gamma$-secretase ( $\mathrm{Li}$ et al., 2000; Esler et al., 2000). However, biochemical evidence showing that the transition-state analogs directly target the aspartates in TMD6 and TMD7 is lacking. A helical peptide-based inhibitor mimicking the TMD structure binds to PS fragments but does not compete with the binding of transition-state analog inhibitors (Das et al., 2003; Kornilova et al., 2005). Likewise, the dipeptidic inhibitor DAPT binds to PS1 CTF in yet a third manner, distinct from transition-state analogs and helical peptides (Morohashi et al., 2006). We analyzed the accessibility of substituted cysteines in the presence of $\gamma$-secretase inhibitors [i.e., transition-state analog (L-685,458), helical peptide (pep15), and dipeptidic compound (DAPT)] at concentrations that completely abolish the proteolytic activity of $\gamma$-secretase. Biotinylation of A192C, A398C, and T399C from the luminal side, as well as that of H351C, R377C, G378C, and V379C from the cytosolic side, was not affected by any of the $\gamma$-secretase inhibitors (Fig. 5). In contrast, the accessibility of A246C, L250C, L381C, and L383C, located around the catalytic aspartates, were significantly reduced by L-685,458. pep 15 had no effect on the labeling of any of these residues. Interestingly, DAPT moderately decreased the accessibility of L250C and L383C. It is well established that TMDs of several receptors and transporters rotate to cause structural rearrangements with agonist or antagonist treatment (Horenstein et al., 2001; Loo and Clarke, 2001a). However, we did not observe labeling of additional residues originally inaccessible to MTSEA-biotin in the fully inhibited $\gamma$-secretase (data not shown). Moreover, none of the inhibitors affected the biotinylation of A260C and I387C, located in close proximity to the catalytic residues D257 and D385, respectively, and facing the hydrophilic environment. These data suggest that binding of the inhibitors does not induce a significant rotation of TMD6 or
TMD7. Altogether, the inhibition of labeling of A246C, L250C L381C, and L383C by L-685,458 strongly supports the notion that these residues line the pore of the catalytic center together with D257 and D385. In contrast, A260 and I387 appear to locate in a geometrically different milieu from the catalytic pore.

\section{TMD6 and TMD7 of PS1 are in close proximity and face the same catalytic pore}

Although both D257 in NTF and D385 in CTF are required for intramembrane proteolysis, it still remains unclear how many presenilins and aspartates are involved in cis (i.e., catalytic site consisting of D257-D257 or D385-D385 pairs) and/or in trans (catalytic site of D257-D385 pair) during the proteolytic process (Schroeter et al., 2003; Cervantes et al., 2004). To gain insights into the structural characteristics of the catalytic pore, we performed cross-linking experiments using MTS cross-linkers. M4M (MTS-4-MTS) and M6M (MTS-6-MTS) are sulfhydryl-tosulfhydryl cross-linking reagents with spacer moieties of 7.8 and $10.4 \AA$ long, respectively (Loo and Clarke, 2001b). We chose to examine the in cis cross-linking of two cysteines introduced to residues L250, L383, or I387. Cross-linking experiments using microsome fractions revealed that neither of the cross-linkers caused a robust shift in band pattern of holoprotein, NTF, or CTF of the single-Cys mt PS1 (Fig. 6). We next generated a double-Cys mt PS1 harboring a pair of cysteine residues, located in NTF and CTF, respectively, in a single molecule (i.e., L250C/L383C, $\mathrm{L} 250 \mathrm{C} / \mathrm{I} 387 \mathrm{C}$ ). These mutants retained $\gamma$-secretase activity (supplemental Fig. $1 C$, available at www.jneurosci.org as supplemental material). MTS cross-linkers did not affect the banding pattern of L250C/L383C. In contrast, coincubation of L250C/I387C with MTS cross-linkers significantly decreased the amount of NTF and CTF, and a band close in size to that of a holoprotein $(\sim 45 \mathrm{kDa})$ emerged. This band reacted with both anti-PS1 Nand C-terminal antibodies but not with antibodies to other $\gamma$-secretase components (data not shown), suggesting that this polypeptide corresponds to an NTF-CTF heterodimer. The decrease in the molecular weight of this cross-linked NTF-CTF heterodimer compared with that of holoprotein might be as a result of a truncation, heterogeneity at the $\mathrm{N}$ terminus of CTF (Podlisny et al., 1997), and/or to a structural change in intrapacking of TMDs, which has been reported in cross-linking experiments (Loo and Clarke, 2000; Tolia et al., 2006). Supporting the latter possibility, the amount of holoprotein was decreased after cross-linking, whereas no holoprotein-dimer was observed. These data indicate that TMD6 and TMD7 are facing the same hydrophilic pore and that L250 and I387 are closely located (i.e., $\leq 7.8 \AA$ apart).

\section{Discussion}

In this study, we applied SCAM in combination with competition assays (Fig. 7A) to a fine structural analysis of the catalytic pore of $\gamma$-secretase, and showed the following: (1) HR6 and HR8 of PS1 span the membrane as TMD6 and TMD7, respectively; (2) the catalytic aspartates reside within a hydrophilic, funnel-shaped pore connected to both the cytosolic and extracellular sides; and (3) the cytosolic portion of TMD7, encompassing the conserved GxGD motif, is highly water accessible, which is not characteristic of an $\alpha$-helix structure within the membrane, and likely forms a hydrophilic active site within the pore (Fig. 7C). Recently, Tolia et al. (2006) reported on a SCAM analysis of PS1 showing that some substituted cysteines in TMD6 and TMD7 were accessible to $N$-[6-(biotinamido)hexyl]-3' -(2'-pyridyldithio)-propionamide (EZ-link biotin-HPDP). However, substitutions were limited to 
conserved residues and loss of function PS1 mutants (G382A, G384C, and D385C) that are liable to significant structural changes were also included in the analysis. Indeed, they showed that G384C and D385C were reactive to biotin-HPDP, although we did not observe a significant biotinylation of these inactive mutants either by MTSEA-biotin or biotin-HPDP (supplemental Fig. 3, available at www. jneurosci.org as supplemental material). We cannot exclude the possibility that the different chemistry of the reagents and the difference in the constructs may affect the labeling results. Most importantly, the competition experiments using charged reagents and inhibitors are vital to the topography and functional significance of the biotinylated residues. In this regard, our present data provide important mechanistic implications on how the $\gamma$-secretase complex hydrolyzes transmembrane substrates; PS1 forms a catalytic pore with a unique structure within the lipid bilayer.

\section{TMD6 of PS1 in an active}

$\gamma$-secretase complex

From the extracellular side of TMD6, A246 and L250 were water-accessible residues with restrictions, suggesting that these residues are located inside a narrow crevice within the membrane. Together with the lack of biotinylation of A260C from the luminal side, we speculate that TMD6 is facing a funnel-shaped "catalytic pore" tapered around the catalytic aspartate D257. Moreover, A246 and L250 are located on the same interface of TMD6 in line with the catalytic D257, based on the $\alpha$-helical model of a single-span TMD

(Fig. $7 B$ ). Considering that the labeling of these residues was inhibited by L-685,458, it is strongly suggested that the $\mathrm{N}$-terminal half of TMD6 serves as a binding site for this compound on $\gamma$-secretase. This hypothesis is supported by our recent TMD-swap studies showing that the preservation of the luminal half of TMD6 is required for the $\gamma$-secretase activity, but not for the formation of the high-molecular-weight complex (Watanabe et al., 2005). In contrast, cysteines introduced into the cytosolic side of TMD6, except for A260C, were not labeled. Moreover, the labeling of A260C was not affected by any of the $\gamma$-secretase inhibitors. This suggests that the geometrical localization of the cytosolic half of TMD6 relative to the hydrophilic pore is different from that of the active site (Fig. 7C). Notably, we confirmed the labeling of W247C, which is located on a different interface from the catalytic D257 in the $\alpha$-helical model (Tolia et al., 2006), by EZ-link biotin-HPDP, thus supporting the hypothesis that TMD6 is facing a hydrophilic cavity different from the catalytic pore (C. Sato, T. Tomita, and T. Iwatsubo, unpublished observations). Collectively, our data suggest that TMD6 has a kinked structure tapered around the catalytic center and that the luminal half of it is directly involved in the formation of the pore wall and in substrate cleavage as a subsite.

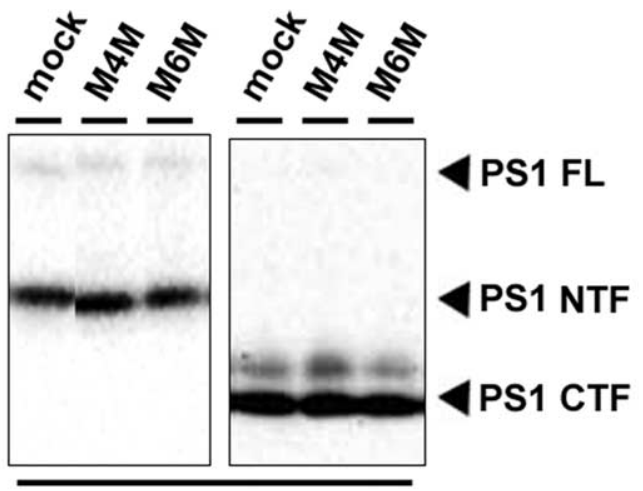

L250C

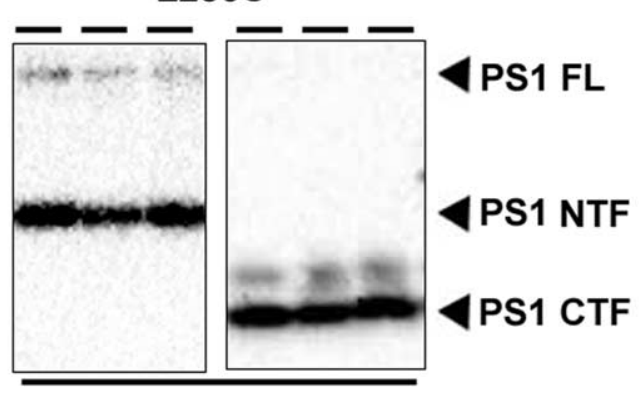

L250C/L383C

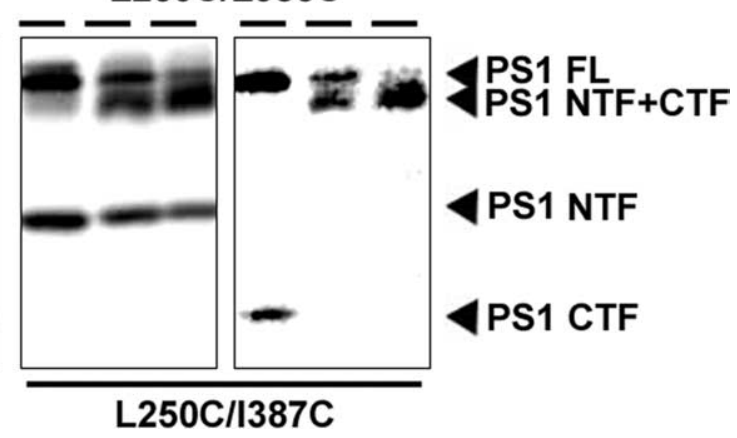

Figure 6. Cross-linking experiment using MTS cross-linkers. Thiol-mediated cross-linking experiments of single-Cys or doubleCys mt in TMD6 (L250C) and TMD7 (L383C and I387C) using MTS cross-linkers are shown.

TMD7 of PS1 in an active $\boldsymbol{\gamma}$-secretase complex

Our SCAM analysis confirmed the widely accepted topological model of PS1 wherein HR8 penetrates the membrane as TMD7 from the cytosol to the luminal side (Fig. 7A). Notably, the labeling pattern of cysteine residues introduced into TMD7 was totally distinct between the cytosolic and luminal sides: the former is highly hydrophilic, whereas the latter is hydrophobic. Most importantly, consecutive residues of the cytosolic side appeared to reside within a pore-like, narrow aqueous environment, suggesting that this region, including the GXGD motif, is forming a hydrophilic structure within the pore and not simply a membrane-embedded $\alpha$-helix. The hydrophilic structure comprising a part of a TMD located within an aqueous pore is highly reminiscent of the "plug and pore" structure of the SecY complex (Van den Berg et al., 2004). The SecY complex is a protein translocation channel with a large funnel-like cavity. The bottom of the channel is blocked by a hydrophilic plug consisting of a part of TMD2 of SecY, which regulates the opening of the pore. Here we speculate that the cytosolic side of TMD7 forms a plug-like structure containing an active site ("catalytic plug") embedded in the catalytic pore that is tapered around D385 (Fig. 7C). This could explain why HR8 is highly hydrophilic, albeit it penetrates the 

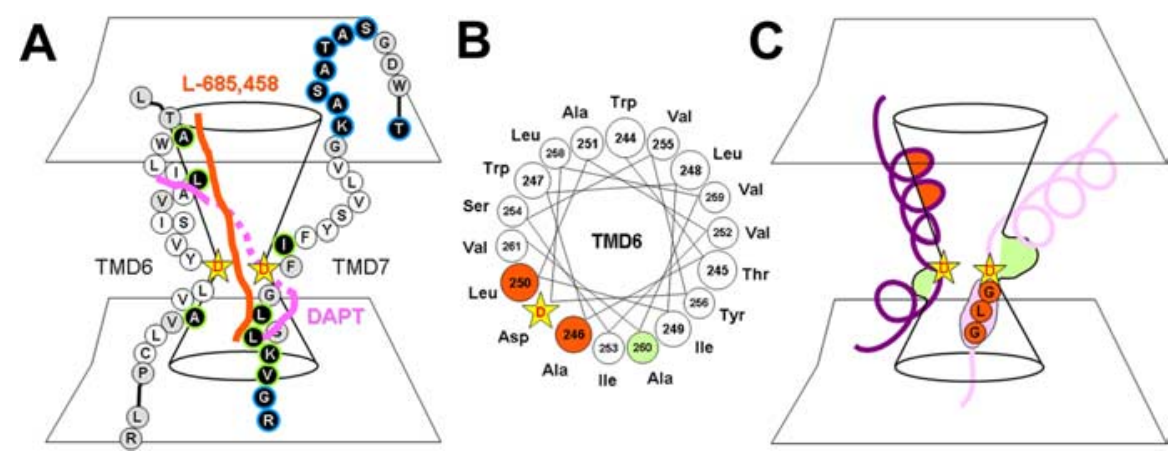

Figure 7. Hypothetical structure around the catalytic site of $\gamma$-secretase. A, A summary of SCAM analysis. Catalytic aspartates are indicated by yellow stars. Cysteine mutants that were labeled by MTSEA-biotin are shown by a white letter in a black circle. The labeling of residues that were effectively competed by MTSES or MTSET is shown in a blue frame, and the unaffected residues are in a green frame. Residues that were not labeled by MTSEA-biotin or unanalyzed are shown by a black letter in a white and gray circle, respectively. Putative locations of L-685,458 and DAPT within the catalytic pore of PS1 are indicated by orange and pink lines, respectively. $\boldsymbol{B}, \alpha$-Helical wheel alignment consisting of the 18 amino acids of TMD 6 of human PS1 starting at the N-terminal tryptophan 244. Catalytic aspartate at position 257 is indicated by a star. Residues that were biotin labeled and competed by L-685,458 (i.e., A246 and L250) are shown by orange circles. A260 was indicated by a green circle. Note that A246 and L250 are aligned on the same interface with D257 in an $\alpha$-helical model. C, Schematic depiction of the configuration of the catalytic pore. TMD6 and TMD7 are shown in purple and pink lines, respectively. Catalytic aspartates are shown in yellow stars. Regions that serve as subsites, including the conserved motif forming a catalytic site (pink oval), are indicated in orange. Residues that are close to the aspartates but putatively facing a geometrically different hydrophilic environment from the catalytic pore are shown in green.

membrane. The recent finding that L383 of PS1 determines the substrate specificity of $\gamma$-secretase is consistent with our model, because $\mathrm{L} 383$ locates at a different interface from the active site if the catalytic motif were to form an $\alpha$-helical structure (Yamasaki et al., 2006). Indeed, the structural model that a hydrophilic pore in the TMD harbors an active site also fits well with other I-CLiPs. For example, SREBP (sterol regulatory element binding protein) site 2 protease (S2P) has a zinc-binding HEXXH catalytic motif within hydrophobic residues (Zelenski et al., 1999). Rhomboid family proteases harbor a highly conserved GASGG motif that locates within a predicted TMD in a manner remarkably similar to other traditional serine proteases (Freeman, 2004). If these catalytic motifs were involved in an $\alpha$-helical structure, several conserved residues that are known as essential residues to proteolysis in classical proteases would be located on a different interface of TMD. We hypothesize that the active site within the hydrophilic pore is a structure common to a wide range of I-CLiPs. To further verify this model, a fine structural analysis at atomic level using $\mathrm{x}$-ray crystallography, or other innovative techniques, would be required.

None of the residues at the luminal half of TMD7, except for I387, were labeled with MTSEA-biotin, suggesting that this region is not directly involved in the formation of a water-accessible catalytic pore. This portion of TMD7 may be responsible for the maintenance of the normal active conformation of PS1. Alternatively, these residues may be facing a different pore or crevice that is not accessible from the extracellular space. The finding that the labeling of I387C is not affected by any of the inhibitors but is in close proximity to L250C as determined by cross-linking supports the view that the catalytic pore faces a different hydrophilic milieu that is not directly involved in catalysis. In summary, our data suggest that TMD7 of PS1 functions as a catalytic site in the hydrophilic pore but is not directly involved in the formation of the funnel-like catalytic pore, implying that other TMDs participate in the formation of the wall of the water-accessible pore.

\section{Implications of the catalytic pore structure to the mechanism of intramembrane proteolysis}

Chemical biological analyses using inhibitors as a molecular probe shed light on the molecular mechanism of intramembrane cleavage by $\gamma$-secretase. In this study, we showed that L-685,458 directly inhibited the labeling of A246C and L250C in PS1 NTF, as well as that of L381C and L383C in PS1 CTF. These data suggest that the transition-state analog inhibitors directly target the catalytic aspartates within TMD6 and TMD7 and that A246, L250, L381, and L383 are positioned in line with the catalytic aspartates within the aqueous milieu. The cross-linking result of two cysteines introduced to L250 and I387 supports the idea that PS1 NTF and CTF are located in close proximity and face toward the same hydrophilic pore. Thus, our data suggest that the two catalytic aspartates cooperatively and simultaneously target the scissile bond of a hydrophobic substrate within the catalytic pore structure.

In contrast, DAPT affected only the labeling of L250C and L383C. Consistent with this, chemical biological analyses revealed that DAPT photoprobe DAP-BpB directly binds to PS1 CTF and this DAPT binding site is not identical to but overlaps with that of transitionstate analogs (Morohashi et al., 2006). Thus, the DAPT binding site may be located around L383 that comprises the catalytic site, overlapping with a subsite in PS1 NTF. Finally, the helical peptide-type inhibitor pep15 (Das et al., 2003) had no effect on the biotinylation of any mutants, suggesting that the labeling competition by L-685,458 and DAPT was a specific phenomenon. However, photoprobes derived from the helical peptide bound to PS1 NTF as well as to CTF (Kornilova et al., 2005), suggesting that the helical peptide targets to a PS1 NTF/CTF interface different from the catalytic pore formed by TMD6 and TMD7. Determination of the binding sites of inhibitors by protein biochemical analysis will address this issue. Nonetheless, SCAM analyses combined with inhibitor competition studies provide significant mechanistic insights into the intramembrane proteolysis. Additional information on the fine structure of the active $\gamma$-secretase provided by SCAM as well as by other structural analyses will shed light on the molecular mechanism whereby TMDs are recognized as substrates, transferred to and cleaved within the catalytic pore of the $\gamma$-secretase complex in the membrane.

\section{References}

Akabas MH, Kaufmann C, Cook TA, Archdeacon P (1994) Amino acid residues lining the chloride channel of the cystic fibrosis transmembrane conductance regulator. J Biol Chem 269:14865-14868.

Cervantes S, Saura CA, Pomares E, Gonzalez-Duarte R, Marfany G (2004) Functional implications of the presenilin dimerization: reconstitution of $\gamma$-secretase activity by assembly of a catalytic site at the dimer interface of two catalytically inactive presenilins. J Biol Chem 279:36519-36529.

Das C, Berezovska O, Diehl TS, Genet C, Buldyrev I, Tsai JY, Hyman BT, Wolfe MS (2003) Designed helical peptides inhibit an intramembrane protease. J Am Chem Soc 125:11794-11795.

Doan A, Thinakaran G, Borchelt DR, Slunt HH, Ratovitsky T, Podlisny M, Selkoe DJ, Seeger M, Gandy SE, Price DL, Sisodia SS (1996) Protein topology of presenilin 1. Neuron 17:1023-1030. 
Esler WP, Kimberly WT, Ostaszewski BL, Diehl TS, Moore CL, Tsai JY, Rahmati T, Xia W, Selkoe DJ, Wolfe MS (2000) Transition-state analogue inhibitors of $\gamma$-secretase bind directly to presenilin-1. Nat Cell Biol 2:428-434.

Freeman M (2004) Proteolysis within the membrane: rhomboids revealed. Nat Rev Mol Cell Biol 5:188-197.

Haass C, Steiner H (2002) Alzheimer disease $\gamma$-secretase: a complex story of GxGD-type presenilin proteases. Trends Cell Biol 12:556-562.

Hayashi I, Urano Y, Fukuda R, Isoo N, Kodama T, Hamakubo T, Tomita T, Iwatsubo T (2004) Selective reconstitution and recovery of functional $\gamma$-secretase complex on budded baculovirus particles. J Biol Chem 279:38040-38046.

Herreman A, Serneels L, Annaert W, Collen D, Schoonjans L, De Strooper B (2000) Total inactivation of $\gamma$-secretase activity in presenilin-deficient embryonic stem cells. Nat Cell Biol 2:461-462.

Horenstein J, Wagner DA, Czajkowski C, Akabas MH (2001) Protein mobility and GABA-induced conformational changes in $\mathrm{GABA}(\mathrm{A})$ receptor pore-lining M2 segment. Nat Neurosci 4:477-485.

Kaback HR, Sahin-Toth M, Weinglass AB (2001) The kamikaze approach to membrane transport. Nat Rev Mol Cell Biol 2:610-620.

Kan T, Tominari Y, Morohashi Y, Natsugari H, Tomita T, Iwatsubo T, Fukuyama T (2003) Solid-phase synthesis of photoaffinity probes. Highly efficient incorporation of biotin-tag and cross-linking groups. Chem Commun (Camb) 7:2244-2245.

Karlin A, Akabas MH (1998) Substituted-cysteine accessibility method. Methods Enzymol 293:123-145.

Kornilova AY, Bihel F, Das C, Wolfe MS (2005) The initial substratebinding site of $\gamma$-secretase is located on presenilin near the active site. Proc Natl Acad Sci USA 102:3230-3235.

Kuner T, Wollmuth LP, Karlin A, Seeburg PH, Sakmann B (1996) Structure of the NMDA receptor channel M2 segment inferred from the accessibility of substituted cysteines. Neuron 17:343-352.

Laudon H, Hansson EM, Melen K, Bergman A, Farmery MR, Winblad B, Lendahl U, von Heijne G, Naslund J (2005) A nine-transmembrane domain topology for presenilin 1. J Biol Chem 280:35352-35360.

Lazarov VK, Fraering PC, Ye W, Wolfe MS, Selkoe DJ, Li H (2006) Electron microscopic structure of purified, active $\gamma$-secretase reveals an aqueous intramembrane chamber and two pores. Proc Natl Acad Sci USA 103:6889-6894.

Li X, Greenwald I (1996) Membrane topology of the C. elegans SEL-12 presenilin. Neuron 17:1015-1021.

Li X, Greenwald I (1998) Additional evidence for an eight-transmembranedomain topology for Caenorhabditis elegans and human presenilins. Proc Natl Acad Sci USA 95:7109-7114.

Li YM, Xu M, Lai MT, Huang Q, Castro JL, DiMuzio-Mower J, Harrison T, Lellis C, Nadin A, Neduvelil JG, Register RB, Sardana MK, Shearman MS, Smith AL, Shi XP, Yin KC, Shafer JA Gardell SJ (2000) Photoactivated $\gamma$-secretase inhibitors directed to the active site covalently label presenilin 1. Nature 405:689-694.

Loo TW, Clarke DM (2000) The packing of the transmembrane segments of human multidrug resistance P-glycoprotein is revealed by disulfide crosslinking analysis. J Biol Chem 275:5253-5256.

Loo TW, Clarke DM (2001a) Cross-linking of human multidrug resistance P-glycoprotein by the substrate, tris-(2-maleimidoethyl)amine, is altered by ATP hydrolysis. Evidence for rotation of a transmembrane helix. J Biol Chem 276:31800-31805.

Loo TW, Clarke DM (2001b) Determining the dimensions of the drugbinding domain of human P-glycoprotein using thiol cross-linking compounds as molecular rulers. J Biol Chem 276:36877-36880.

Morohashi Y, Kan T, Tominari Y, Fuwa H, Okamura Y, Watanabe N, Sato C, Natsugari H, Fukuyama T, Iwatsubo T, Tomita T (2006) C-terminal fragment of presenilin is the molecular target of a dipeptidic $\gamma$-secretasespecific inhibitor DAPT $(N$-[N-(3,5-difluorophenacetyl)-L-alanyl $]-S$ phenylglycine $t$-butyl ester). J Biol Chem 281:14670-14676.

Ogura T, Mio K, Hayashi I, Miyashita H, Fukuda R, Kopan R, Kodama T,
Hamakubo T, Iwastubo T, Tomita T, Sato C (2006) Three-dimensional structure of the $\gamma$-secretase complex. Biochem Biophys Res Commun 343:525-534.

Podlisny MB, Citron M, Amarante P, Sherrington R, Xia W, Zhang J, Diehl T, Levesque G, Fraser P, Haass C, Koo EH, Seubert P, St George-Hyslop P, Teplow DB, Selkoe DJ (1997) Presenilin proteins undergo heterogeneous endoproteolysis between Thr291 and Ala299 and occur as stable Nand C-terminal fragments in normal and Alzheimer brain tissue. Neurobiol Dis 3:325-337.

Roberts DD, Lewis SD, Ballou DP, Olson ST, Shafer JA (1986) Reactivity of small thiolate anions and cysteine- 25 in papain toward methyl methanethiosulfonate. Biochemistry 25:5595-5601.

Schroeter EH, Ilagan MX, Brunkan AL, Hecimovic S, Li YM, Xu M, Lewis HD, Saxena MT, De Strooper B, Coonrod A, Tomita T, Iwatsubo T, Moore CL, Goate A, Wolfe MS, Shearman M, Kopan R (2003) A presenilin dimer at the core of the $\gamma$-secretase enzyme: insights from paralle analysis of Notch 1 and APP proteolysis. Proc Natl Acad Sci USA 100:13075-13080.

Seal RP, Amara SG (1998) A reentrant loop domain in the glutamate carrier EAAT1 participates in substrate binding and translocation. Neuron 21:1487-1498.

Seal RP, Leighton BH, Amara SG (1998) Transmembrane topology mapping using biotin-containing sulfhydryl reagents. Methods Enzymol 296:318-331

Seal RP, Leighton BH, Amara SG (2000) A model for the topology of excitatory amino acid transporters determined by the extracellular accessibility of substituted cysteines. Neuron 25:695-706.

Steck TL, Weinstein RS, Straus JH, Wallach DFH (1970) Inside-out red cel membrane vesicles: preparation and purification. Science 168:255-257.

Takasugi N, Tomita T, Hayashi I, Tsuruoka M, Niimura M, Takahashi Y, Thinakaran G, Iwatsubo T (2003) The role of presenilin cofactors in the $\gamma$-secretase complex. Nature 422:438-441.

Tolia A, Chavez-Gutierrez L, De Strooper B (2006) Contribution of presenilin transmembrane domains 6 and 7 to a water-containing cavity in the $\gamma$-secretase complex. J Biol Chem 281:27633-27642.

Tomita T, Iwatsubo T (2006) $\gamma$-Secretase as a therapeutic target for treatment of Alzheimer's disease. Curr Pharm Des 12:661-670.

Tomita T, Maruyama K, Saido TC, Kume H, Shinozaki K, Tokuhiro S, Capell A, Walter J, Grunberg J, Haass C, Iwatsubo T, Obata K (1997) The presenilin 2 mutation (N141I) linked to familial Alzheimer disease (Volga German families) increases the secretion of amyloid $\beta$ protein ending at the 42nd (or 43rd) residue. Proc Natl Acad Sci USA 94:2025-2030.

Tomita T, Takikawa R, Koyama A, Morohashi Y, Takasugi N, Saido TC, Maruyama K, Iwatsubo T (1999) C terminus of presenilin is required for overproduction of amyloidogenic A $\beta 42$ through stabilization and endoproteolysis of presenilin. J Neurosci 19:10627-10634.

Van den Berg B, Clemons Jr WM, Collinson I, Modis Y, Hartmann E, Harrison SC, Rapoport TA (2004) X-ray structure of a protein-conducting channel. Nature 427:36-44.

Watanabe N, Tomita T, Sato C, Kitamura T, Morohashi Y, Iwatsubo T (2005) Pen-2 is incorporated into the $\gamma$-secretase complex through binding to transmembrane domain 4 of presenilin 1. J Biol Chem 280:41967-41975.

Wilson GG, Karlin A (1998) The location of the gate in the acetylcholine receptor channel. Neuron 20:1269-1281.

Wolfe MS (2006) The $\gamma$-secretase complex: membrane-embedded proteolytic ensemble. Biochemistry 45:7931-7939.

Yamasaki A, Eimer S, Okochi M, Smialowska A, Kaether C, Baumeister R, Haass C, Steiner H (2006) The GxGD motif of presenilin contributes to catalytic function and substrate identification of $\gamma$-secretase. J Neurosci 26:3821-3828.

Zelenski NG, Rawson RB, Brown MS, Goldstein JL (1999) Membrane topology of S2P, a protein required for intramembranous cleavage of sterol regulatory element-binding proteins. J Biol Chem 274:21973-21980. 